\title{
The accuracy of Raman spectroscopy in the diagnosis of lung cancer: a systematic review and meta-analysis
}

\author{
Cong Chen ${ }^{1,2 \#}$, Jianqi Hao ${ }^{1,2 \#}$, Xiaohu Hao ${ }^{1,2 \#}$, Wenying Xu ${ }^{2}$, Congjia Xiao ${ }^{1}$, Jian Zhang ${ }^{1}$, Qiang Pu ${ }^{1}$, Lunxu Liu ${ }^{1}$ \\ ${ }^{1}$ Department of Thoracic Surgery, Institute of Thoracic Oncology, West China Hospital, Sichuan University, Chengdu, China; ${ }^{2}$ West China School \\ of Medicine, Sichuan University, Chengdu, China \\ Contributions: (I) Conception and design: L Liu, J Hao; (II) Administrative support: Q Pu, L Liu; (III) Provision of study materials or patients: C \\ Chen, X Hao; (IV) Collection and assembly of data: W Xu, J Zhang; (V) Data analysis and interpretation: C Chen, X Hao; (VI) Manuscript writing: \\ All authors; (VII) Final approval of manuscript: All authors. \\ \#These authors contributed equally to this work. \\ Correspondence to: Prof. Lunxu Liu, MD, PhD, FRCS. No. 37, Guoxue Alley, Chengdu, 610041, China. Email: lunxu_liu@aliyun.com.
}

Background: To investigate the overall performance of Raman Spectroscopy (RS) in the diagnosis of lung cancer.

Methods: We systematic searched databases including PubMed, EMBASE, CNKI and Web of science for studies up to May 2020 with no start date limited. Then we extracted data of true positives, true negatives, false positives and false negatives from the included studies to calculate the pooled sensitivity, specificity, accuracy, positive and negative likelihood ratios (LRs), and diagnostic odds ratio (DOR), with $95 \%$ confidence intervals to evaluate the diagnostic value of Raman spectroscopy. We plotted the summary receiver operator characteristics (SROC) and the area under the curve (AUC) to evaluate the overall performance of Raman spectroscopy. Quality assessments and Publication bias were evaluated by QUADAS-2 checklist and Stata software version 12.0.

Results: Totally, 12 studies were included in our meta-analysis. The pooled diagnostic sensitivity and specificity of Raman Spectroscopy in lung cancer were 0.90 (95\% CI, 0.87-0.92, P<0.05) and 0.76 (95\% CI, 0.72-0.79, $\mathrm{P}<0.05$ ). The pooled PLR and NLR were 5.87 (95\% CI, 3.45-9.97) and 0.14 (95\% CI, 0.10-0.22) respectively. And the AUC of SROC curve was 0.9453.

Discussion: Raman spectroscopy had excellent accuracy with high sensitivity and considerable specificity in the diagnosis of lung cancer.

Keywords: Raman Spectroscopy; lung cancer; meta-analysis

Submitted Mar 22, 2021. Accepted for publication Jun 25, 2021.

doi: $10.21037 /$ tcr-21-515

View this article at: https://dx.doi.org/10.21037/tcr-21-515

\section{Introduction}

Lung cancer is a primary malignant tumor of mucosa or glands arising from the trachea or bronchus. The recent decades, lung cancer was always rank at the top of the list by the morbidity and mortality rate among most of the cancers, with a low five-year survival rate between $10-18 \%$ all over the world (1-5). Early diagnosis and treatment of lung cancer can make a considerable improvement in the therapeutic effect and prognosis of lung cancer patients.
Routine diagnostic method includes physical examination, imaging examination and biopsy. However, each of these approaches has its drawbacks, such as lower sensitivity or specificity, time-consuming, or inescapable invasiveness (6). Subsequently, faster, more convenient and minimize invasive diagnostic tools with a high sensitivity and specificity are urgently needed to be applied in the field of early lung cancer diagnosis.

Raman spectroscopy, which analyses biological or chemical substances based on the scattered spectrum 
associated with vibrational and rotational information of molecular structures, has been widely used as an analytical tool in many clinical fields. Several studies have shown that Raman spectroscopy can provide high specificity and sensitivity for cancer research and detect the changes in biomolecules so as to differentiate and locate the normal and mutated tissue and cells $(7,8)$. Compared with routine diagnostic methods, Raman spectroscopy is an effective diagnostic tool with minimal sample preparation, destructiveness and invasiveness. It has advantages in high sensitivity and specificity, detection of macromolecules and compatible in physiological measurements. Moreover, it also performed well in vivo fiber-optic applications, chemical analysis, quantification, classification and imaging of biological samples (9). On the one hand, improved technologies based on Raman spectroscopy have been emerging such as Raman micro-spectroscopy, surfaceenhanced Raman scattering (SERS), and near infrared Raman spectra (NIR-SERS) in recent years which made the detection result of Raman spectroscopy more accurate and reliable (10-12). On the other hand, Raman scattering has an impressive performance in detecting and differentiating samples from various sources such as tissues, liquid or dried blood serum/plasma with the broadened application of Raman spectroscopy $(4,10,13)$.

Although several single-center original studies have been conducted recently to detect the diagnostic value in the diagnosis of lung cancer by using Raman spectroscopy, different experimental design, sample acquisition method and selection of Raman spectroscopy technology in each center made the pool result ambiguous. Therefore, these studies cannot effectively reveal the value of Raman spectrum in the diagnosis of lung cancer due to their own limitations. Aimed to comprehensively evaluate the overall performance of Raman Spectroscopy in the diagnosis of lung cancer, we performed this meta-analysis and systematic review to investigate the diagnostic value of Raman Spectroscopy. We present the following article in accordance with the Cochrane Handbook for Systematic Reviews of Intervention (version 5.1.0) and the PRISMA reporting checklist (14) (available at https://dx.doi.org/10.21037/tcr-21-515).

\section{Methods}

\section{Literature research}

Two reviewers independently searched the databases including PubMed, EMBASE, CNKI and Web of science for studies up to May 2020 with no start date limited. The primary search was performed with the combination of the relevant medical subject heading $(\mathrm{MeSH})$ terms, key words and variations for "lung", "cancer" and "Raman spectra". We had no restrictions on language or research type in the primary search. Discussion was conducted to resolve the inconsistencies in the process of primary search. We also screened the reference lists of relevant primary studies and review articles to make an addition to our eligible articles.

\section{Selection criteria and exclusion criteria}

We systematic screened all the articles from the primary search and carefully selected the eligible articles that met our criteria. Studies included should meet the following criteria: (I) Only human tissue should be allowed to use in the trail. (II) Raman spectroscopy was reported as an independent diagnostic tool to identify the lung cancer and differentiate it from normal tissue. (III) The studies should provide fourfold table for true positives (TP), true negatives (TN), false positives (FP) and false negatives (FN) or sensitivity and specificity values which can be calculated. (IV) A control group (healthy samples or samples with other specific diagnoses) should be included in the studies. (V) Articles should be published in English or Chinese.

Exclusion criteria: (I) Studies with nonhuman origin trial were excluded. (II) Irrelevant articles or article types such as reviews, case reports, conference abstracts, letters for articles and editorials were excluded. (III) Studies without providing exact data to calculate, without setting control group, or studies with insufficient samples (the number of spectra samples less than 10) were excluded. Typically, if agreements can't be reached when the discrepancy and discussion happened, we inquired the third reviewer to solve the conflict.

\section{Data extraction}

Two reviews extracted data from included studies independently with a standard tabulation. To avoid bias, the final data tabulation was summarized by comparing the two results, and disagreements were resolved by discussion. The tabulation consists of basic information and final events. The basic information listed as followed: title, author, country, year of publication and baseline indicators (such as stage of lung cancer, mean age, sample type, diagnostic algorithm and the number of samples, etc.). The final events indicators which can reveal the diagnostic value included 
TP, FP, TN, FN, sensitivity and specificity, etc.

\section{Statistical analysis}

The extracted data of TP, FP, TN and FN, the calculation results including the pooled sensitivity, specificity, accuracy, positive and negative likelihood ratios (LRs), and diagnostic odds ratio (DOR), with $95 \%$ CIs were implemented to investigate the diagnostic value of Raman spectra. In addition, we plotted the summary receiver operator characteristics (SROC) curves to assess the relationship between sensitivity and specificity. And the area under the curve (AUC) was calculated to assess the overall performance of Raman spectroscopy. In general, the diagnostic tool is rated as excellent when the AUC value is greater than 0.9; the result is good when the value between 0.8 and 0.9 ; the value shows a fair grade with the result range 0.7 to 0.8 and while less than 0.7 as poor.

All of the above statistical analysis was implemented by using Meta-Disc version 1.4 software.

\section{Quality assessments and publication bias}

We conducted quality assessments for the 12 included studies by using Review Manager 5.3 on the basis of the Quality Assessment of Diagnostic Accuracy Studies (QUADAS-2) checklist. Publication bias were performed with Stata software version 12.0 (STATA Corp, TX, USA) by using Deeks Funnel Plot Asymmetry Test (The appearance of an asymmetrical funnel shape or $\mathrm{P}<0.05$ means the Publication bias exist (15).

\section{Results}

\section{Literature search and selection of studies}

We identified 419 studies from the PubMed, EMBASE, CNKI and Web of science. After removing duplicate studies and screening for titles and abstracts, we screened 42 studies that met the inclusion criteria for full-text reading. Finally, there were 12 studies (1,3-5,16-23) included in the meta-analysis. PRISMA diagram for the study selection is disclosed in Figure 1.

\section{Characteristics, quality assessment and publication bias of the included studies}

The general characteristics of the included studies were presented in the Table 1 . Ten studies $(1,3,5,16,17,19-23)$ were published in English and two studies $(4,18)$ were Chinese. A total of 960 samples were tested in all the studies and 430 patients were included without McGregor's study (17). The spectrum was $785 \mathrm{~nm}$ in 6 studies $(1,5,16-19), 532 \mathrm{~nm}$ in 4 studies $(20-23)$ and $632.8 \mathrm{~nm}$ in 2 studies $(3,4)$. Histopathology was the gold standard of all studies. Principal component analysis (PCA) was the most widely used diagnostic algorithm, which was used in 10 studies (1,3-5,16,18,20-23). Linear discriminate analysis (LDA) was used in 7 studies (3-5,20-23). Other diagnostic algorithms include leave-one out cross-validation (LOOCV), partial least squares-discriminant analysis (PLS-DA), principal components with generalized discriminant analysis (PCGDA) and support vector machine (SVM) were only used in one study. The QUADAS-2 diagram displayed the result of the quality assessment (Figure 2). Most studies conformed to the criteria in QUADAS-2. Due to some literatures couldn't clearly report some items in the quality assessment project, some studies' patient selection and index test items were evaluated as 'unclear' and some literatures' selection of the samples were not random and double-blinded, which may lead to high risk of bias. This may affect the quality of the included literatures. The Deeks' funnel plot asymmetry test indicated that there was no publication bias in these studies (bias $=-0.22, \mathrm{P}=0.827$; Figure 3).

\section{Pooled diagnostic value of Raman spectroscopy in lung cancer}

The sensitivity of the included twelve studies fluctuated between 0.75 and 1 . The sensitivity was more than 0.8 except for two studies $(3,4)$. The pooled sensitivity was $0.90(95 \%$ CI, 0.87-0.92), which meant the Raman Spectroscopy can effectively avoid missed diagnosis (Figure $4 A$ ). The specificity of the studies ranged from 0.61 to 1 . The specificity was more than 0.85 except for two studies $(16,17)$. The pooled specificity was 0.76 (95\% CI, 0.72-0.79), which meant the Raman Spectroscopy could also effectively avoid misdiagnosis (Figure 4B).

Pooled positive LR and negative LR were 5.87 (95\% CI, 3.45-9.97) and 0.14 (95\% CI, 0.10-0.22) respectively. Pooled DOR was 51.35 (95\% CI, 21.83-120.78). Summary receiver operating characteristic curve (SROC curve) was used to evaluated the overall diagnostic efficacy. The area under curve (AUC) of the SROC curve was 0.9453 (Figure 5). 


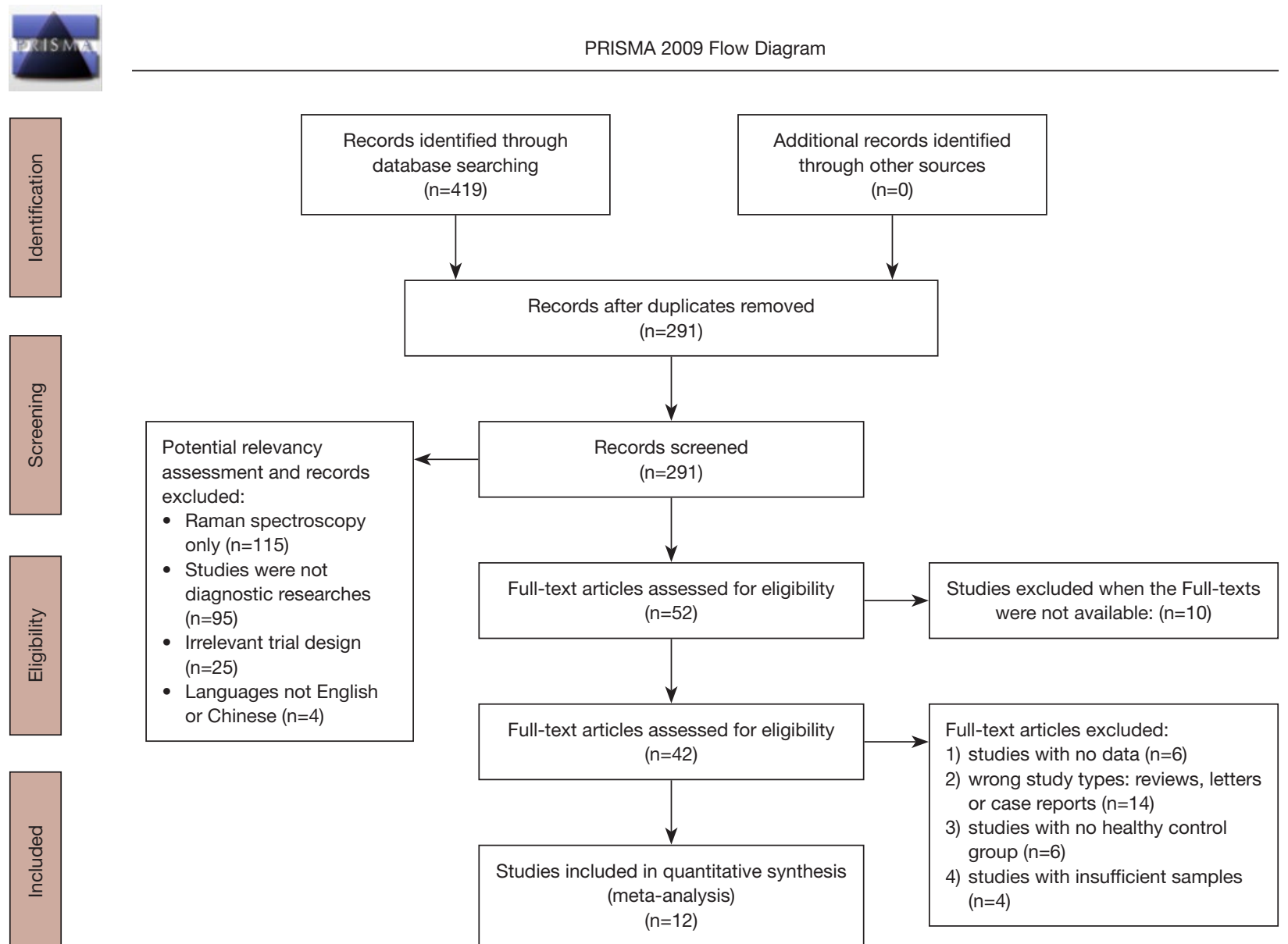

Figure 1 Preferred reporting items for systematic reviews and meta-analyses flowchart.

\section{Subgroup analysis}

In the included studies, three different specimens (lung tissue, blood sample and saliva sample) were used to test. Different types of specimens may have an impact on the accuracy of the test, so we conducted a subgroup analysis of different types of specimens.

\section{Lung tissue}

Lung tissue samples were examined in 5 studies $(1,16,17,22,23)$. The sensitivity of the studies fluctuated between 0.84 and 0.98 . The pooled sensitivity was 0.89 (95\% CI, 0.85-0.93). The specificity of the studies ranged from 0.61 to 1 . The pooled specificity was 0.68 (95\% CI, $0.63-0.73$ ). Pooled PLR and NLR were 3.30 (95\% CI, $1.96-5.58)$ and 0.11 (95\% CI, 0.05-0.25) respectively. Pooled DOR was 50.05 (95\% CI, 11.98-209.18). The AUC of the SROC curve was 0.9878 (Figure 6).

\section{Blood sample}

Blood samples were examined in 4 studies $(5,18,20,21)$. The sensitivity of the studies fluctuated between 0.80 and 0.94. The pooled sensitivity was 0.88 (95\% CI, $0.83-0.93$ ). The specificity of the studies ranged from 0.85 to 0.88 . The pooled specificity was 0.87 (95\% CI, 0.78-0.93). Pooled PLR and NLR were 6.37 (95\% CI, 3.82-10.63) and 0.16 (95\% CI, 0.09-0.26) respectively. Pooled DOR was 40.16 (95\% CI, 18.83-85.65). The AUC of the SROC curve was 0.9302 (Figure 7).

\section{Saliva sample}

Saliva samples were examined in 3 studies $(3,4,19)$. The 


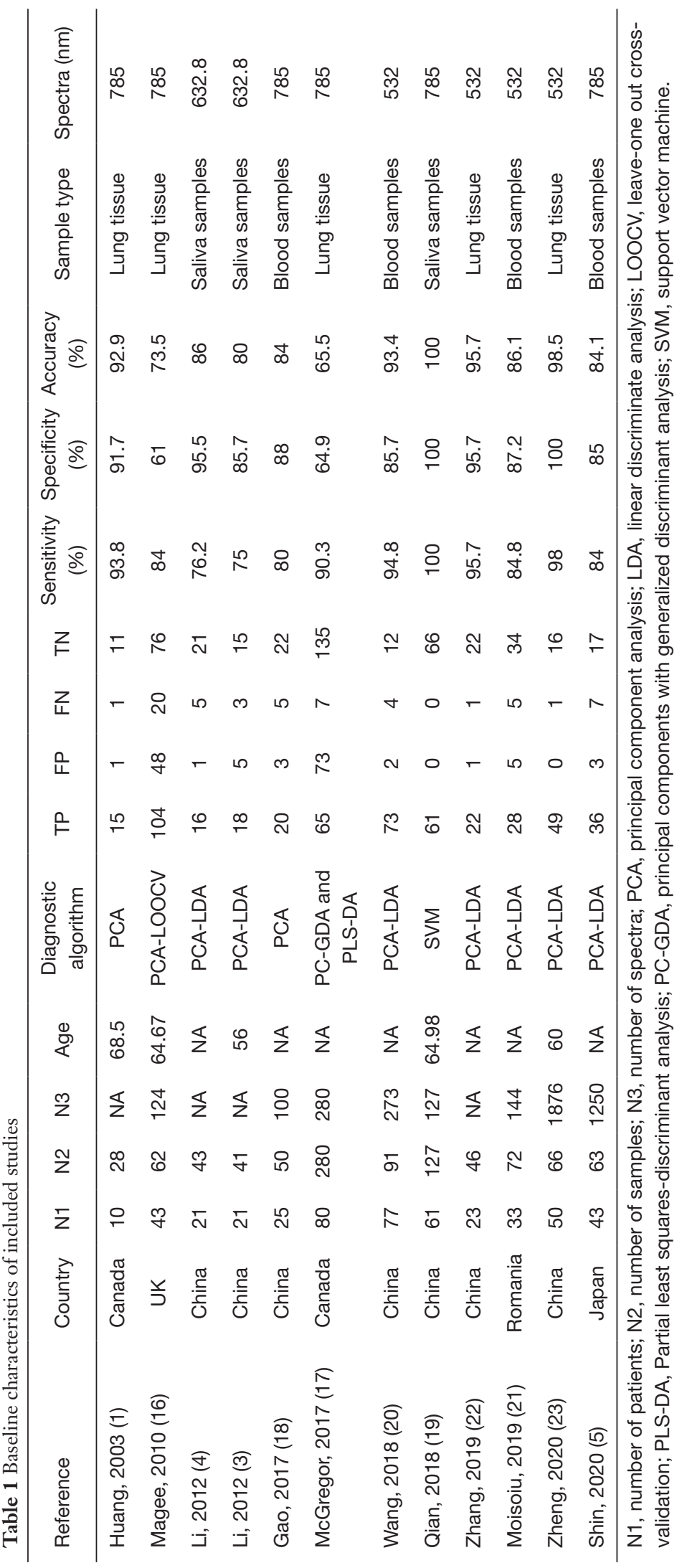



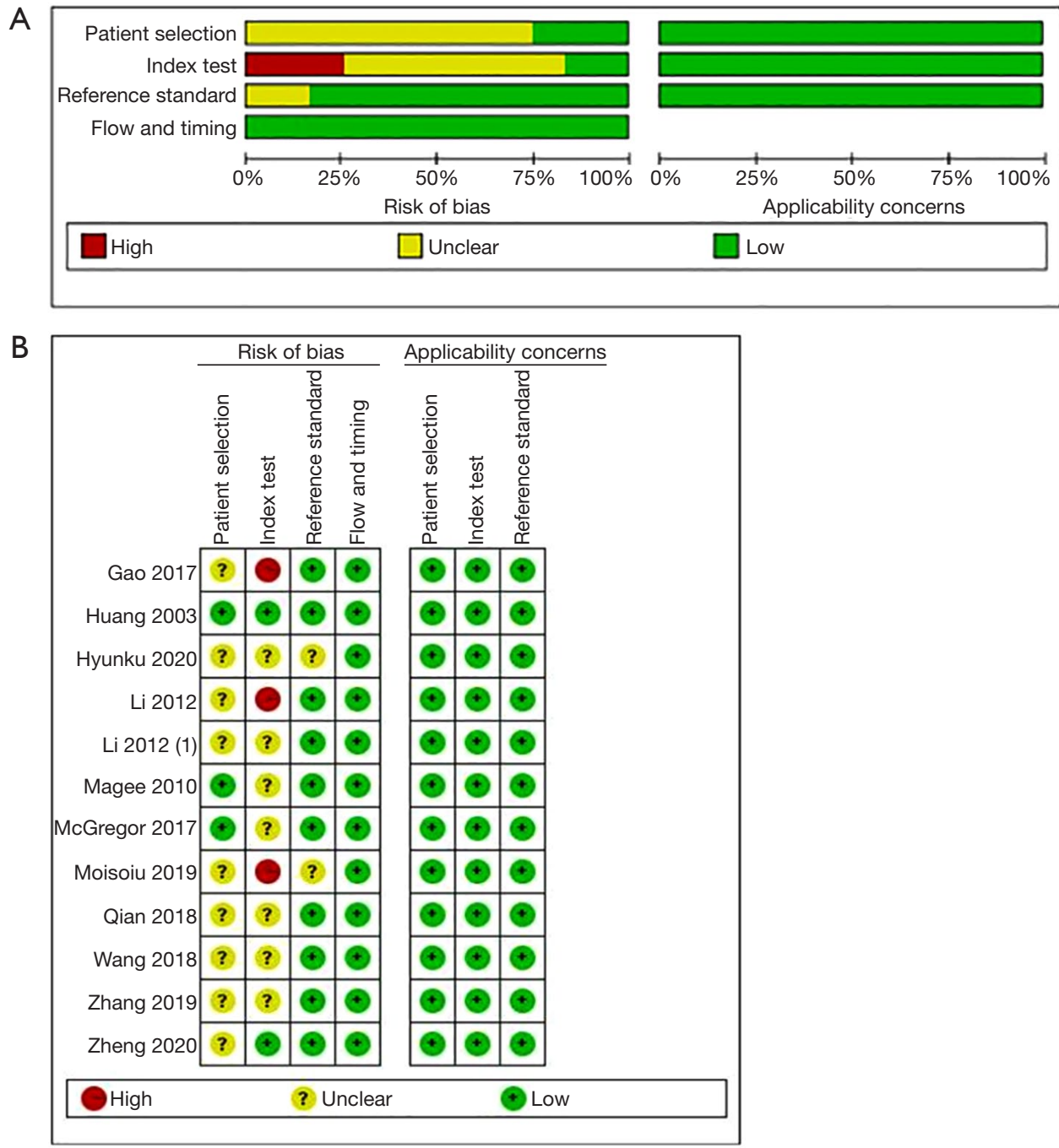

Figure 2 The graphical display of the evaluation of the risk of bias and concerns regarding applicability of the selected studies. (A) Risk of bias and applicability concerns evaluation of included studies in pool. (B) Risk of bias and applicability concerns evaluation of included studies individually.

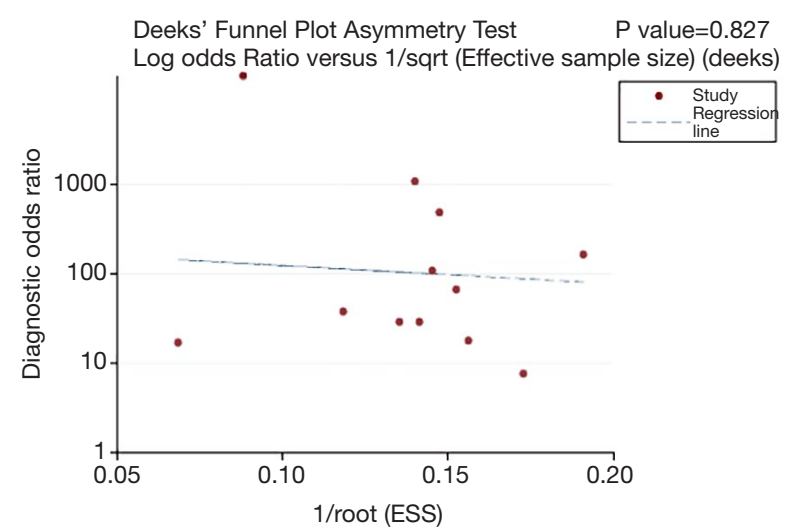

Figure 3 The Deeks' funnel plot asymmetry test indicated that there was no publication bias (bias $=-0.22, \mathrm{P}=0.827$ ). sensitivity of the studies fluctuated between 0.75 and 1 . The pooled sensitivity was 0.92 (95\% CI, 0.85-0.97). The specificity of the studies ranged from 0.85 to 1 . The pooled specificity was 0.94 (95\% CI, 0.88-0.98) (Figure 8). Pooled PLR and NLR were 16.58 (95\% CI, 0.96-287.17) and 0.11 (95\% CI, 0.02-0.67) respectively. Pooled DOR was 170.59 (95\% CI, 6.57-4,431.24). The AUC of the SROC curve was 0.9807 (Figure 8).

\section{Discussion}

Although Raman spectroscopy has been widespread used as a diagnostic tool in the diagnosis of various types of cancer such 

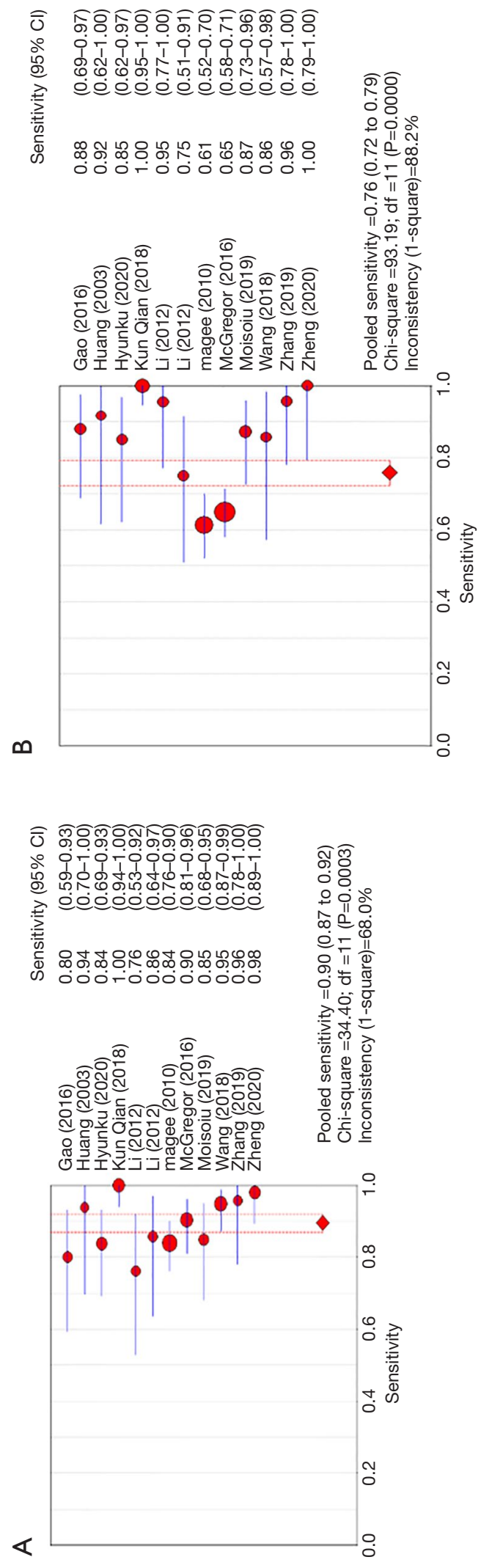

as gastric cancer, breast cancer and skin cancer (24-26), few original clinical trials concerned using Raman spectroscopy to diagnose lung cancer. Therefore, investigations on the overall performance of Raman spectroscopy in the diagnosis of lung cancer was of great significance.

Twelve articles were taken into our meta-analysis. The overall pooled sensitivity and specificity was 0.90 (95\% CI, $0.87-0.92)$ and 0.76 (95\% CI, 0.72-0.79), indicating that Raman spectroscopy had a high identification of lung cancer samples and can distinguish them from normal samples respectively with a low omission diagnostic rate. In addition, the overall pooled DOR was 51.35 (95\% CI, 21.83-120.78) and the AUC was 0.9453 in SROC analysis, suggesting an excellent performance for detecting lung cancer samples by using Raman spectroscopy. Since the differences in sources and methods of obtaining samples may lead to the discrepancies in biological characteristics or levels of signaling molecular hormones among these samples. We divided the included studies into three subgroups, saliva group, lung tissue group and blood group, and the Raman spectral diagnostic efficiency was evaluated and analyzed respectively.

In the subgroup analysis, the pooled sensitivity and specificity of the blood and saliva group were both over 0.85 . And in the lung tissue group, the pooled sensitivity and specificity of lung tissue group were 0.89 and 0.68 . In the included articles of lung tissue group, the results of Raman spectroscopy detection of lung cancer were significantly different and disputed in Magee (16) and McGregor's (17) researches although the results of Raman spectroscopy diagnosis also performed well in their study. In Magee's research, they chose normal samples as a control group from adjacent lung cancer tissue. Although this type of samples was confirmed as "normal" by the pathologists, they might be influenced by signaling molecules expressed during tumor development in adjacent lung cancer tissue, resulting the standard of control group less representative. Question like this also existed in McGregor's study. Samples were divided into two groups: high grade dysplasias (HGDs)/malignant lesions and benign lesions/normal, suggesting that the difference between the two groups may be smaller than that between the theoretical experimental and control groups. Therefore, it wasn't difficult to explain that these two research results both had high sensitivity ( $84 \%$ and $90.3 \%$ respectively), but the lower specificity (61\% and $64.9 \%$ respectively).

According as the physics theory, after a beam of monochromatic light shining on the sample, there are three directions, one part is transmitted, the other is absorbed 

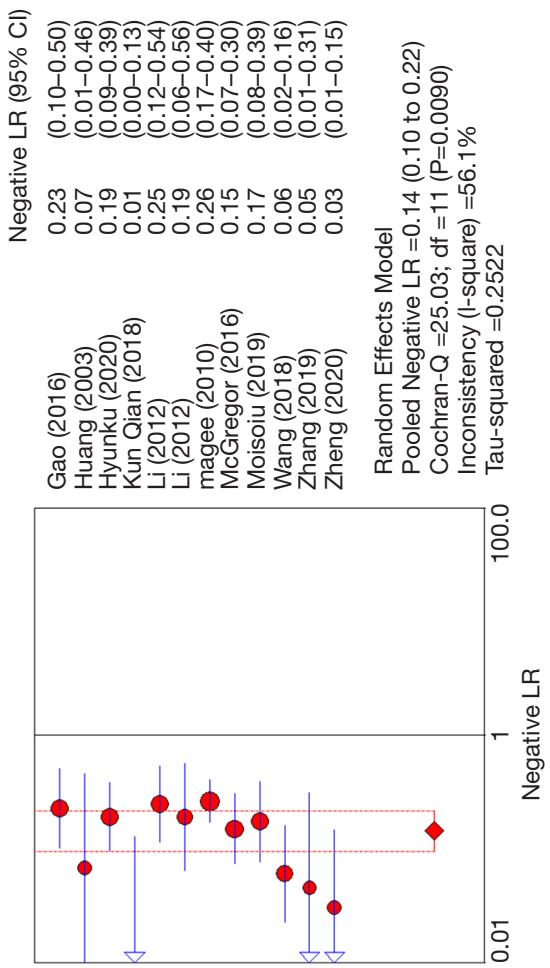

$\infty$

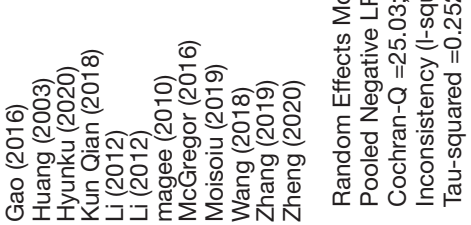

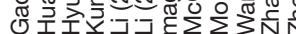

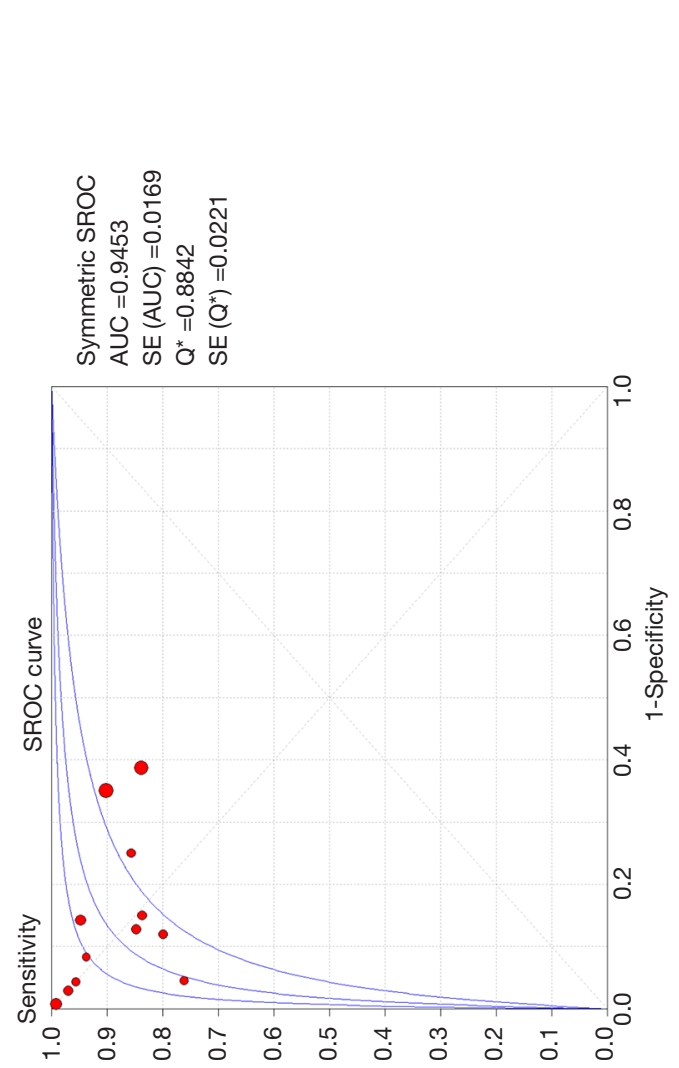

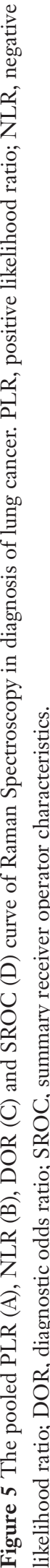
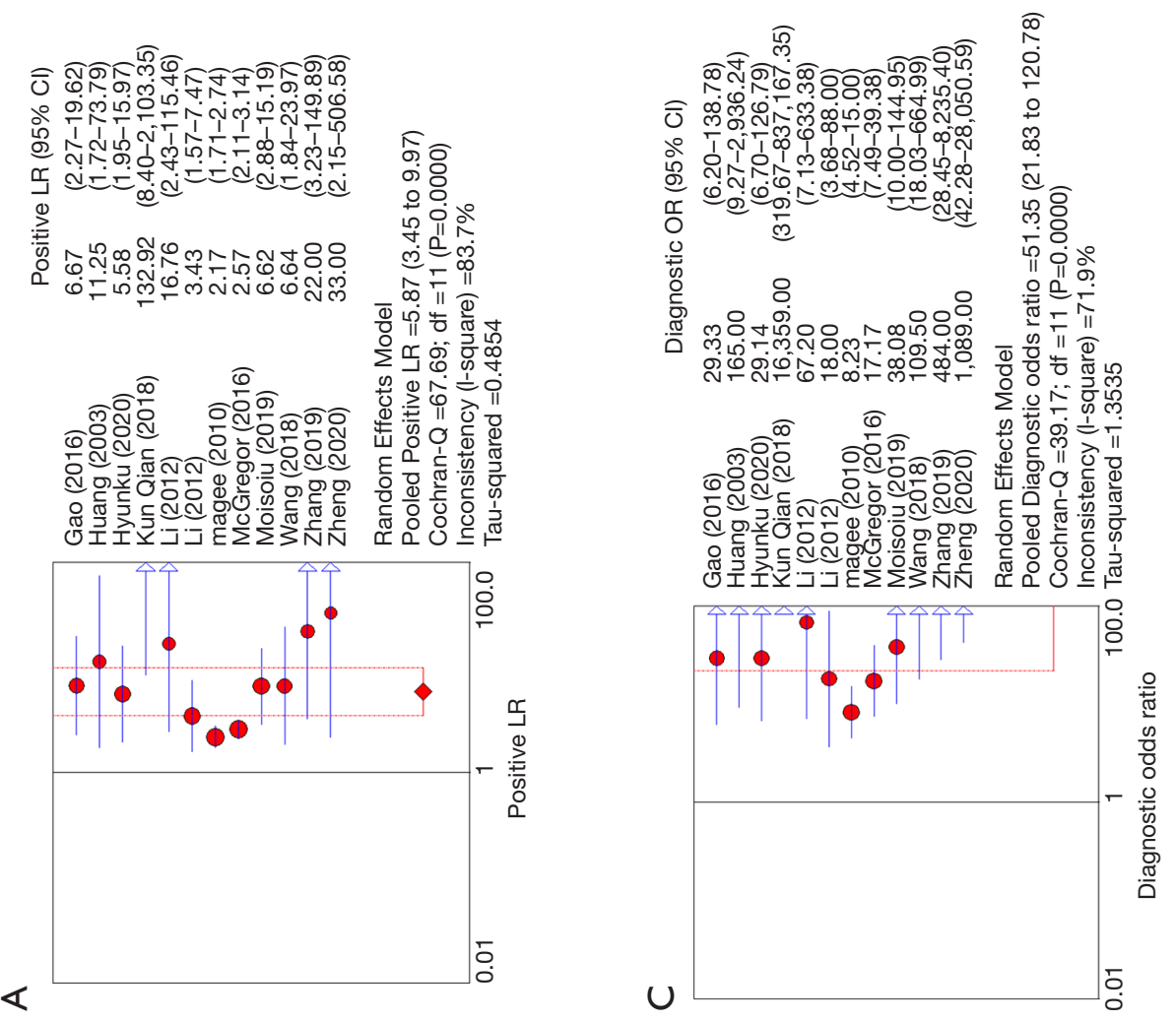

Trransl Cancer Res 2021;10(8):3680-3693 | https://dx.doi.org/10.21037/tcr-21-515 


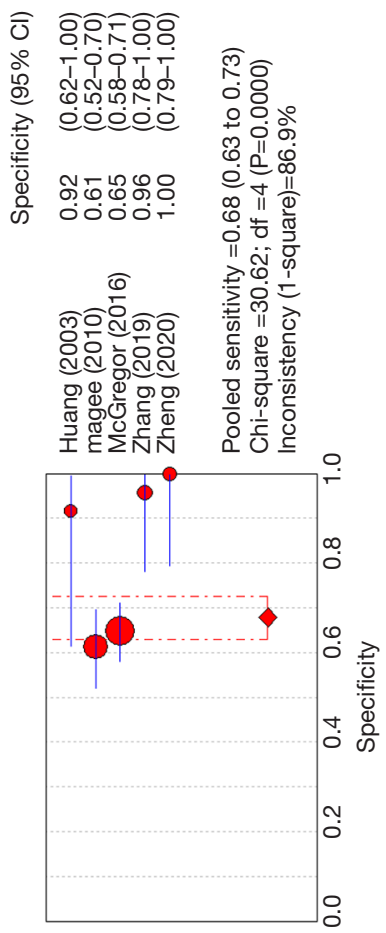

$\infty$

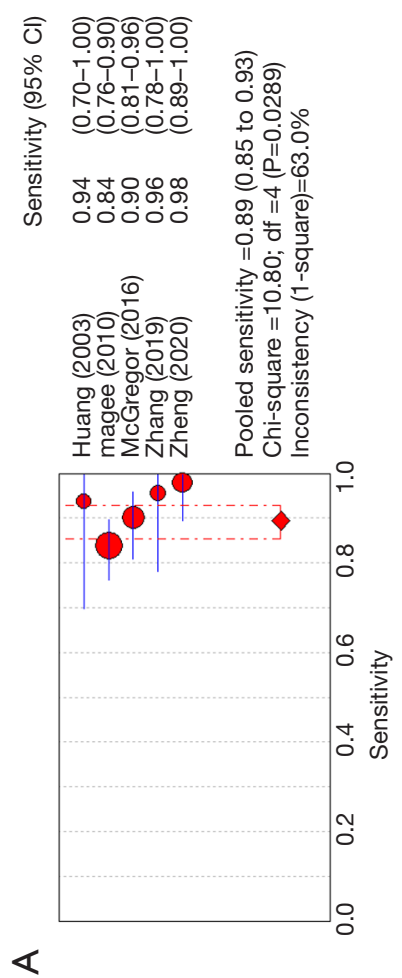

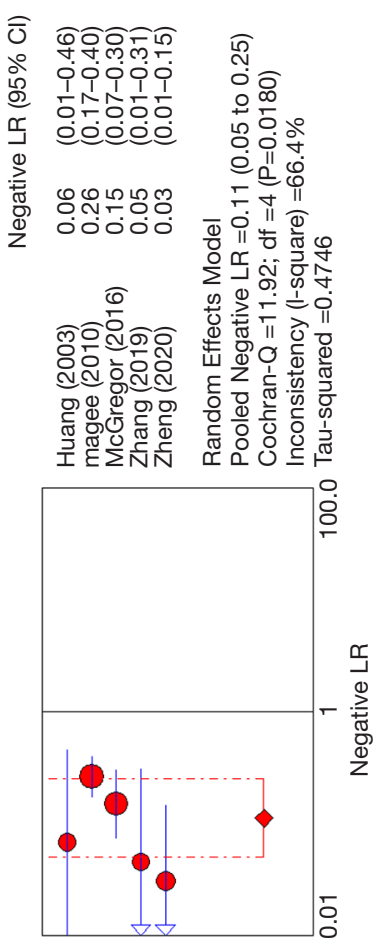

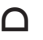
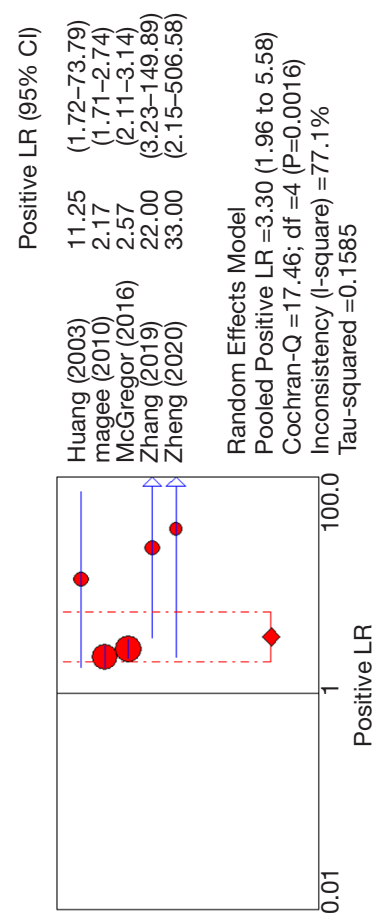

$\cup$

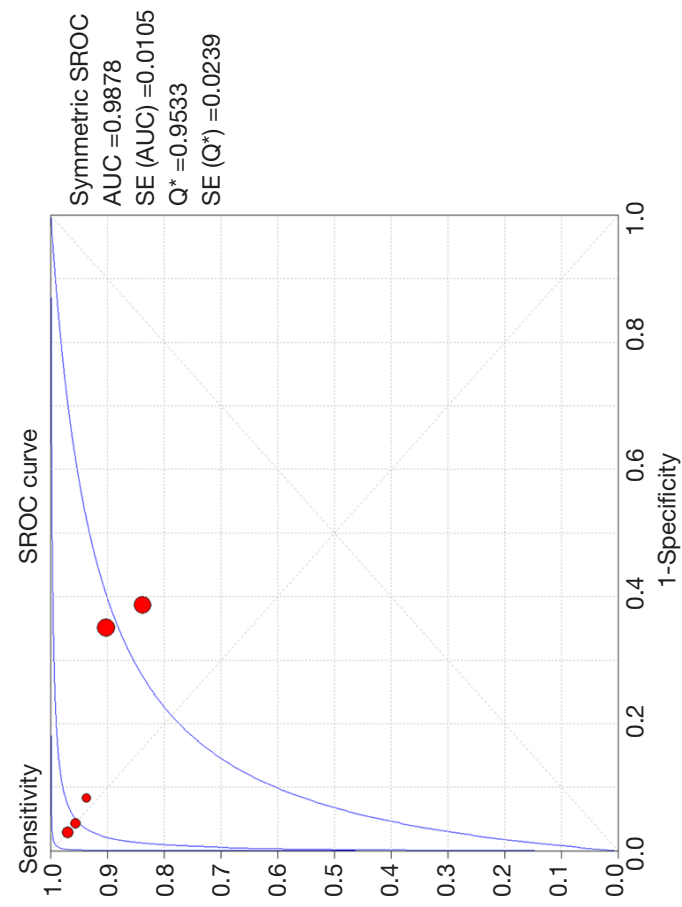

ч
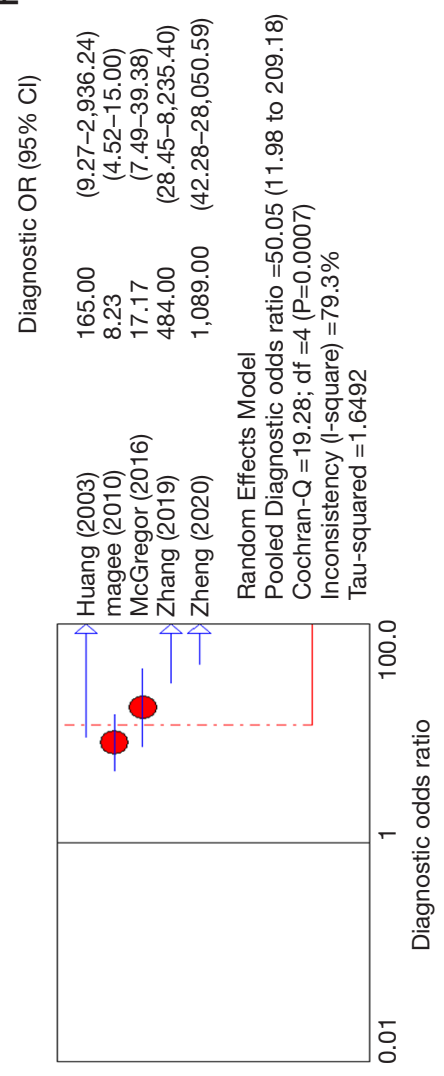

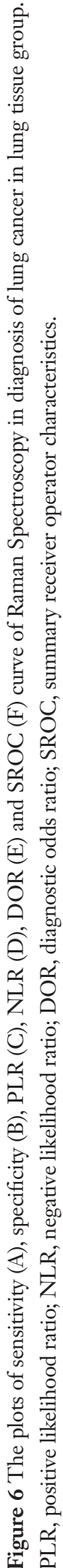




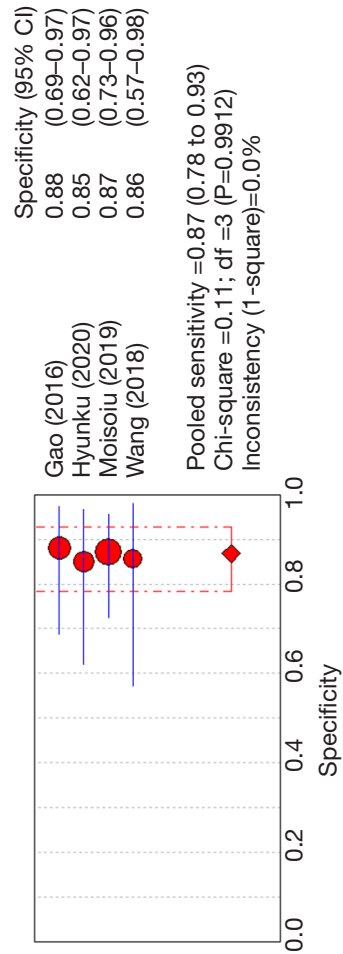

$\infty$

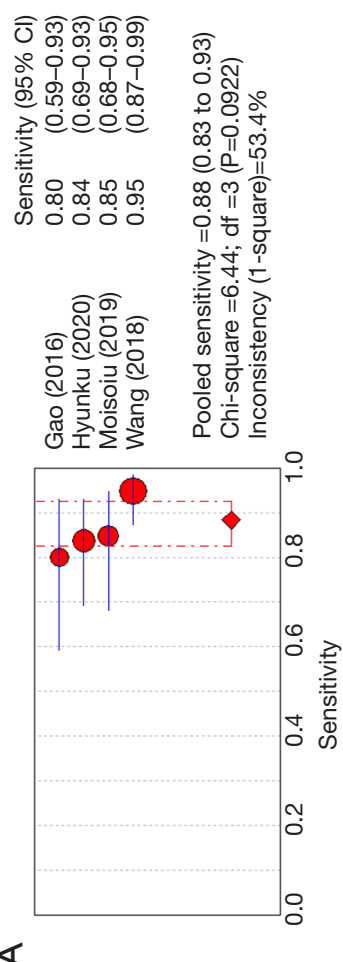

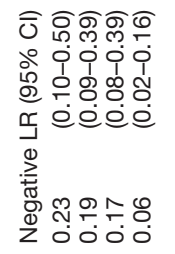

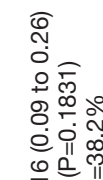

要

는
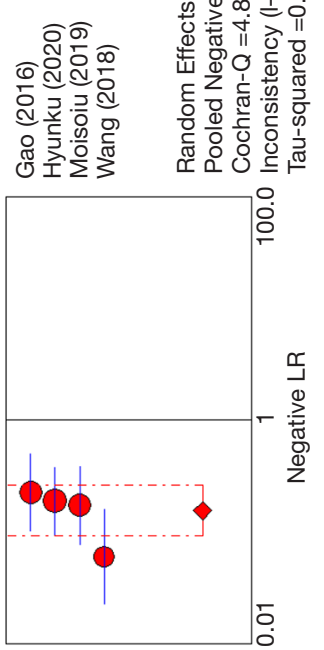

$\triangle$
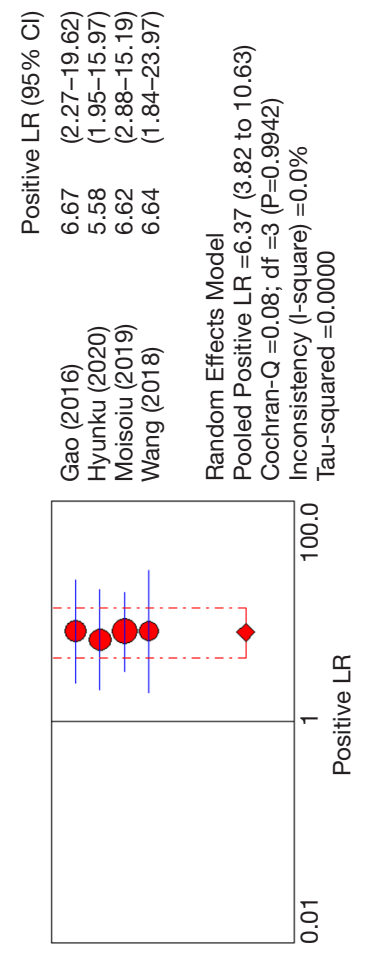

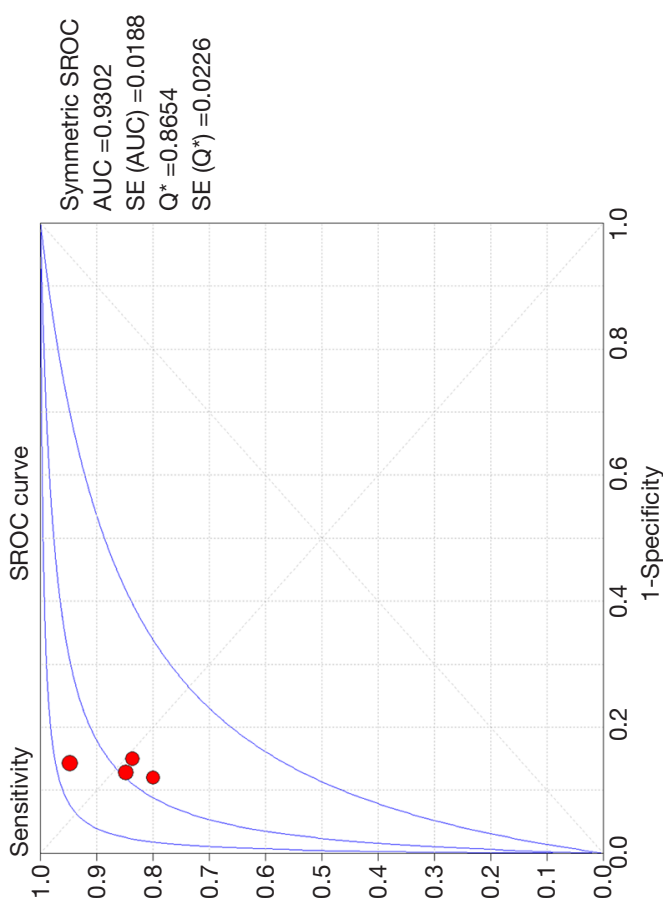



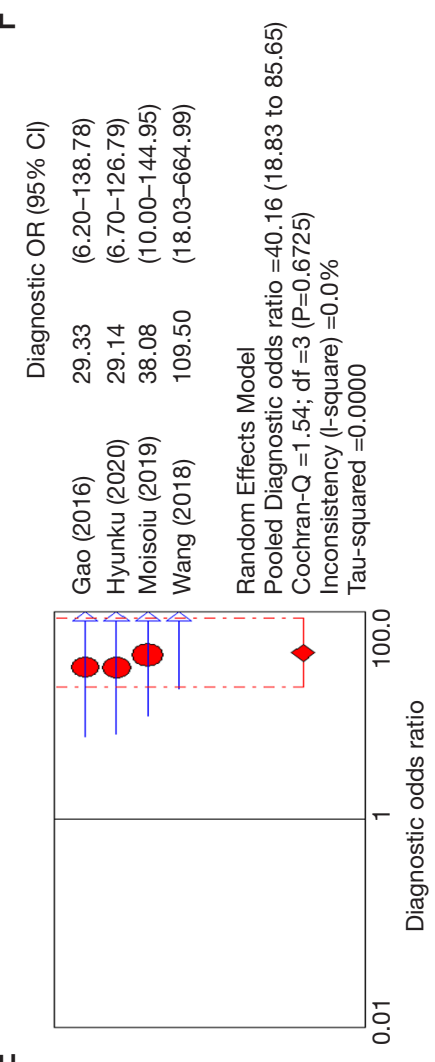

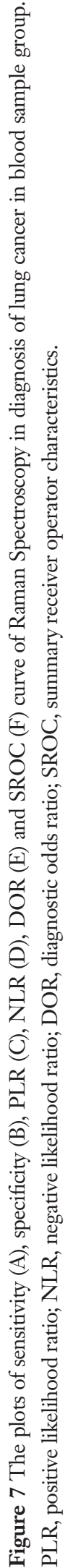




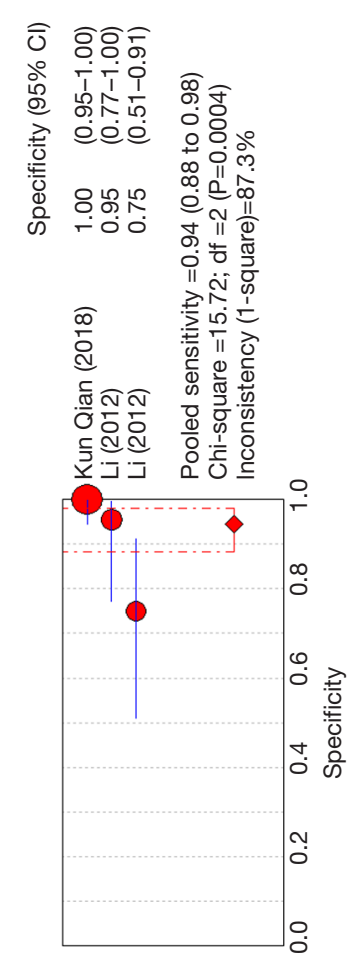

$\infty$

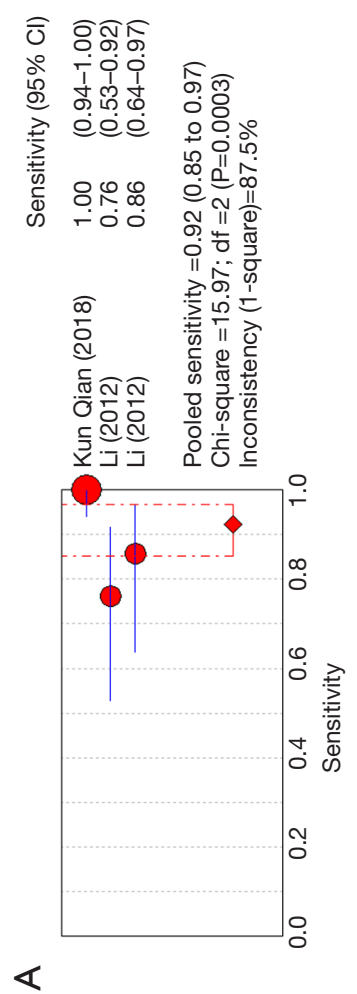

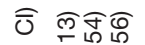
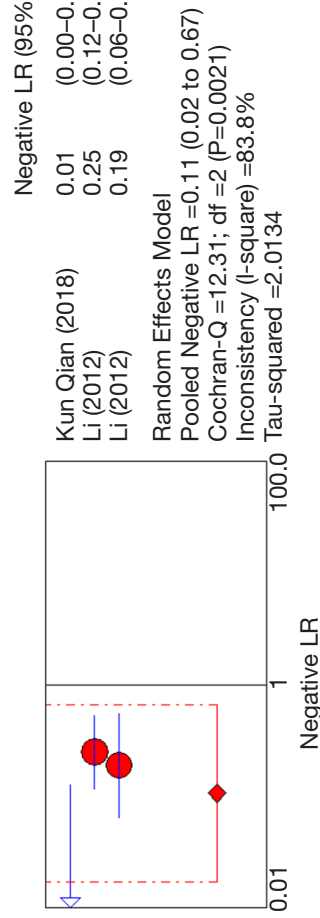

口
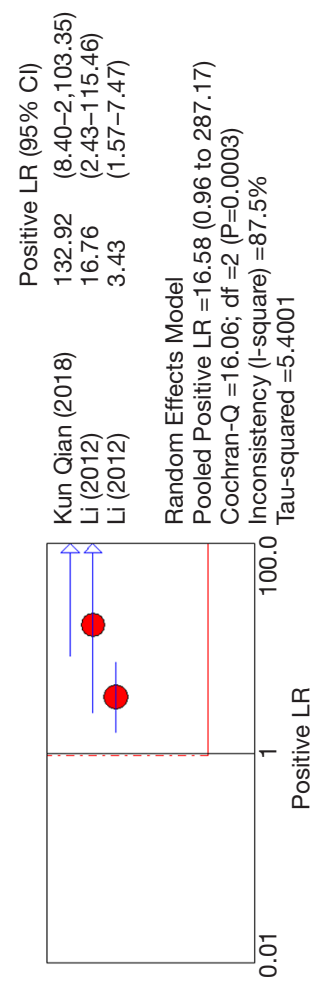

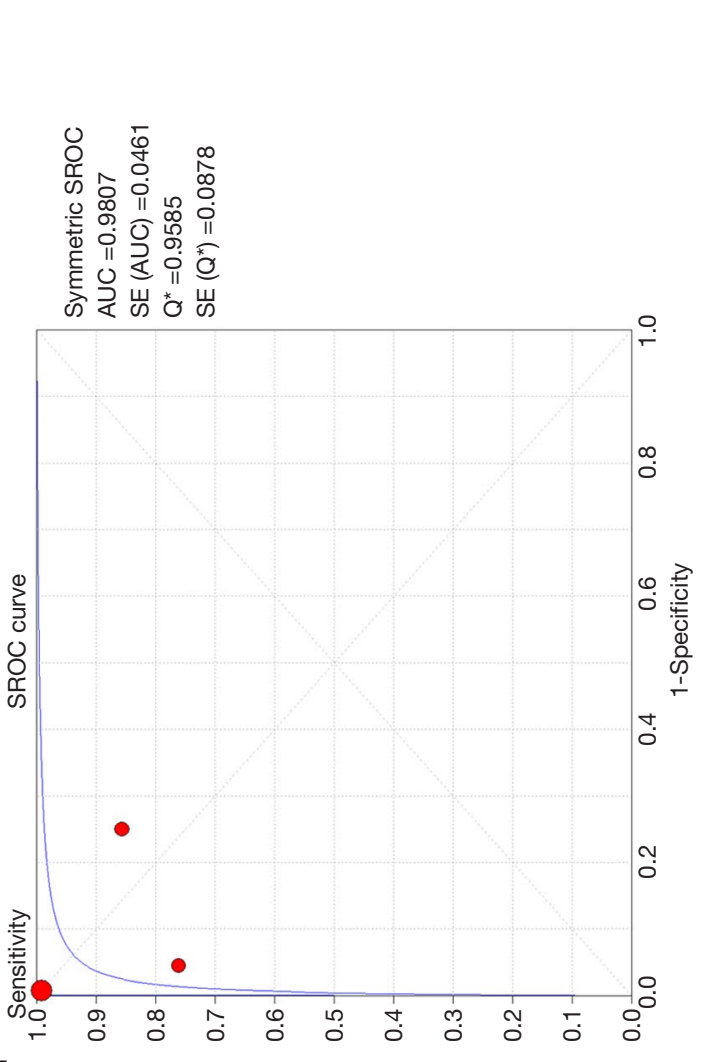

ᄂ
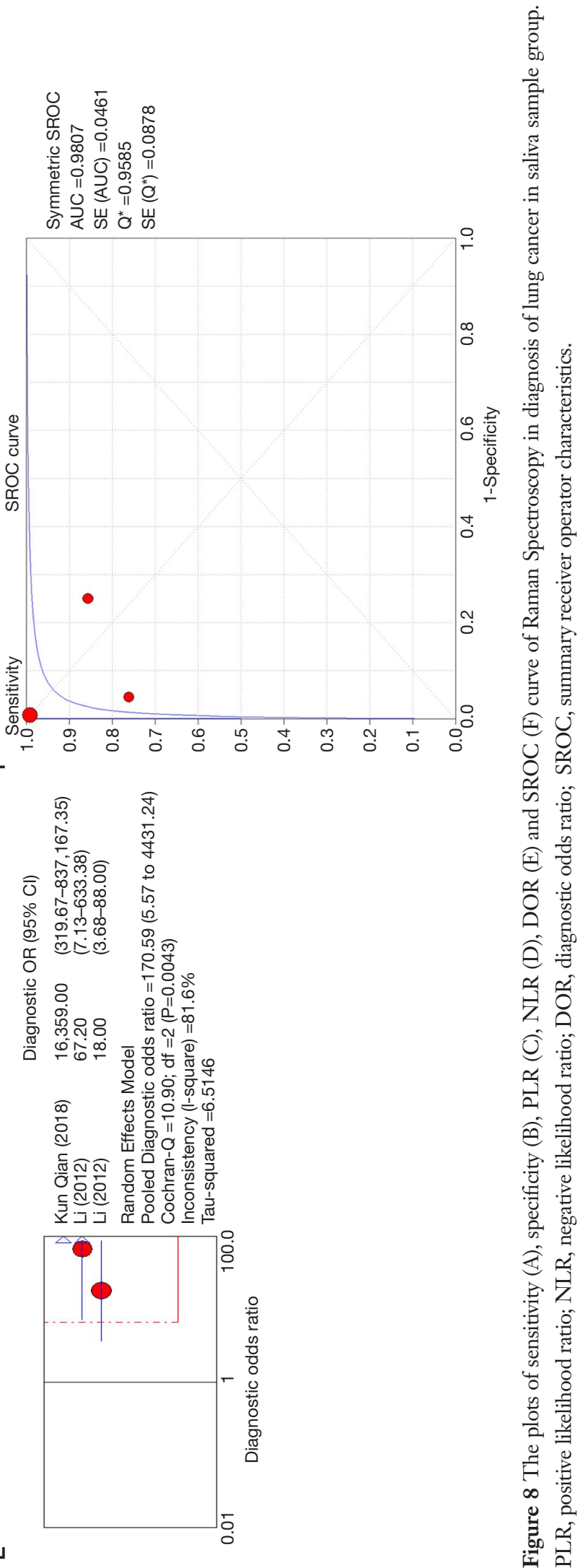

山

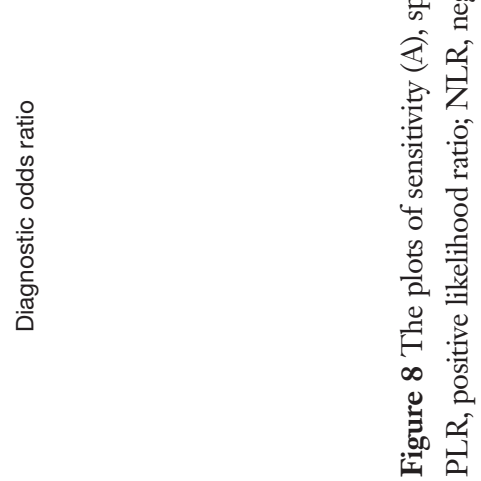


and anther is scattered. Most of the scattered light has the same wavelength as the incident light, however a small part of the scattered light whose wavelength is different from the incident light. The wavelength change is determined by the chemical structure of the sample. This part of the scattering is called Raman scattering (27). Raman spectrum is usually composed of a certain number of Raman peaks. Each Raman peak corresponds to the wavelength position and intensity of Raman scattering light, which corresponds to a specific tissue structure. The diagnosis method based on Raman spectrum usually uses the spectrometer to obtain Raman spectrum from the sample, and then analyzes the spectrum to distinguish the sample. Collecting and classifying the spectra of different tumor tissues is the most direct and extensive application of Raman spectroscopy in the diagnosis of tumor properties. The main focus of Raman spectrum based diagnosis was to find the difference of Raman characteristic peaks between normal samples and cancerous samples (28). The included articles concluded that there were significant differences in peak shift and peak intensity between lung cancer tissue and normal tissue in the spectrum of 785, 532 and $632.8 \mathrm{~nm}$. It suggested the potential application value of Raman spectroscopy in the diagnosis of lung cancer.

Raman spectroscopy not only can be used as a diagnostic tool to identify tumors in various organ systems, but also has the potential to distinguish the various stages of tumor development. Jess et al. (2) performed an experiment with 125 spectra by using Raman spectroscopy to detect five types of cell samples (including normal bronchial epithelial cells (HBEpCs), HBEpCs expressing HPV 16 E7, HBEpCs overexpressing CDK4, BEP2D cell line, AsbTB2A cell line) which represent the neoplastic development in the lung. The results showed that Raman spectroscopy identified abnormal cells with a sensitivity of $91 \%$ and a specificity of $75 \%$, and distinguished between normal cells, cells with extended lifespan (E7 and CDK4 cells) and immortalized/ transformed cells (BEP2D and AsbTB2A) with sensitivities of $75 \%, 79 \%$ and $87 \%$, and specificities of $91 \%, 85 \%$ and $96 \%$. It suggested that Raman spectroscopy can distinguish not only tumor cells and normal cells, but also tumor cells at various stages of development. While the results were encouraging, we also realized the limitations of the study. On the one hand, the normal and tumor cells used in the study were cultured and mutated in vitro. It wasn't clear that Raman spectroscopy can reach the same overall performance when detecting the same type of cell samples from the human body because of the complexity of the human body's hormonal, immune and other regulatory network systems; on the other hand, in the case of lung cancer, more than a dozen types of mutations have been identified, and some types of mutations in lung cancer tissues were not just the combination of several mutations (such as lung adenosquamous carcinoma). Although one purpose of this study was to distinguish the different stages of lung cancer by Raman spectroscopy, the author did not stratify the samples and only divide them into three types of cell lines (including normal cells, cells with extended lifespan and immortalized/transformed cells) which may make the results not widely representative. Therefore, it still needs to be explored by subsequent experiments to find whether the Raman spectrum can be promoted and guarantee a high accuracy in the diagnosis of various types and stages of mutations of lung cancer.

In the progress of the innovation and development of Raman spectroscopy technology for tumor diagnosis, people are constantly trying to figure out how to capture tumor quickly and efficiently while reducing errors and deviations. Hence, techniques such as Raman spectral recognition and diagnosis based on machine learning are emerging. A study about early-stage lung cancer diagnosis by deeplearning based spectroscopic analysis was recently reported. Shin et al. (5) prepared exosomes samples from 20 healthy volunteers and 43 early lung cancer patients with different stages (including 22 stage IA, 16 stage IB, and 5 stage IIB). They developed the deep-learning system to learn how to distinguish the healthy samples from abnormal exosomes samples by utilizing Raman spectroscopy without insufficient human data. And the model turned out to be an accuracy of $95 \%$ for identifying and classifying the exosomes from normal and tumor samples. The study greatly expanded not only the applicable types of Raman spectroscopy materials for lung cancer diagnosis, but also the deep learning as a new technique to improve the accuracy of diagnosis. As the article noted, the exosome was selected as an ideal experimental material because of its purity and abundance in the cancer cells. Due to the molecular characteristics of exosomes, Raman spectrum can only identify the signals from the thick lipid bilayer covering exosomes which were similar with membrane proteins, it may make some confusion with other various membrane proteins during the detecting, leading a misdiagnosis. Another question of the research was the exosomes contained in the samples may derived from other organs or tumor tissues because of the comprehensive source, which can greatly influence the accuracy of the diagnostic results. In addition, fewer sample sources may greatly 
reduce the diversity of data, which makes the applicability of the deep-learning model controversial. Further detailed biochemical analysis and verification experiments based on the combined with several robot learning algorithms are needed to make the deep learn-based Raman spectral diagnosis technology "smarter".

Although our results suggested that Raman spectroscopy can play an important role as a minimally-invasive, high accuracy diagnostic tool in the diagnosis of lung cancer, there were still some limitations in our study. Firstly, despite our study included 4174 Raman spectra from 969 samples, the amounts of samples were small in each research due to the less research in this field. Especially in the group based on humors samples (such as saliva samples), the limited amount of the overall samples can greatly influence the accuracy of Raman spectroscopy. Secondly, we failed to distinguish the stage of the lung cancer samples by using Raman spectroscopy due to the lack recorded of histopathologic staging or other related data from the included studies. Thirdly, as mentioned in the above analysis of the results, some studies [Magee et al. 2010 (16) and McGregor et al. 2017 (17)] have some disputes over the selection and grouping of experimental materials, which affects the overall performance of the Raman spectroscopy. Fourthly, as described in our quality assessments, some articles' selection of the samples were not random and double-blind (such as Li et al. 2012, Li et al. 2012, Qian et al. 2018, Wang et al. 2018, Zhang et al. 2019, Moisoiu et al. 2019, Hyunku et al. Zheng et al. 2020), which may lead to selection bias in the final results. It also reveals that the lack of research in this field leads to the uneven quality of the studies included. Consequently, large sample size, randomized, double-blind original studies are still needed in the future to further verify the diagnostic value of Raman spectroscopy.

\section{Conclusions}

The result of our systematic review and meta-analysis shows that Raman spectroscopy had high sensitivity and considerable specificity for the diagnosis of lung cancer. Raman spectroscopy can be considered as a non-invasive and alternative option for lung cancer diagnosis and has a potential application prospect in the future clinical practice.

\section{Acknowledgments}

Funding: This work was supported by China Postdoctoral
Science Foundation (Grant 2020TQ0210) (ZJ), and the 1.3.5 project for disciplines of excellence, West China Hospital, Sichuan University (Grant ZYGD18021) (LLX).

\section{Footnote}

Reporting Checklist: The authors have completed the PRISMA reporting checklist. Available at https://dx.doi. org/10.21037/tcr-21-515

Peer Review File: Available at https://dx.doi.org/10.21037/ tcr-21-515

Conflicts of Interest: All authors have completed the ICMJE uniform disclosure form (available at https://dx.doi. org/10.21037/tcr-21-515). The authors have no conflicts of interest to declare.

Ethical Statement: The authors are accountable for all aspects of the work in ensuring that questions related to the accuracy or integrity of any part of the work are appropriately investigated and resolved.

Open Access Statement: This is an Open Access article distributed in accordance with the Creative Commons Attribution-NonCommercial-NoDerivs 4.0 International License (CC BY-NC-ND 4.0), which permits the noncommercial replication and distribution of the article with the strict proviso that no changes or edits are made and the original work is properly cited (including links to both the formal publication through the relevant DOI and the license). See: https://creativecommons.org/licenses/by-nc-nd/4.0/.

\section{References}

1. Huang Z, McWilliams A, Lui H, et al. Near-infrared Raman spectroscopy for optical diagnosis of lung cancer. Int J Cancer 2003;107:1047-52.

2. Jess PR, Mazilu M, Dholakia K, et al. Optical detection and grading of lung neoplasia by Raman microspectroscopy. Int J Cancer 2009;124:376-80.

3. Li X, Yang T, Lin J. Spectral analysis of human saliva for detection of lung cancer using surface-enhanced Raman spectroscopy. J Biomed Opt 2012;17:037003.

4. Li XZ, Yang TY, Ding JH. Surface enhanced Raman spectroscopy (SERS) of saliva for the diagnosis of lung cancer. Guang Pu Xue Yu Guang Pu Fen Xi 2012;32:391-3.

5. Shin H, Oh S, Hong S, et al. Early-Stage Lung Cancer 
Diagnosis by Deep Learning-Based Spectroscopic Analysis of Circulating Exosomes. ACS Nano 2020;14:5435-44.

6. Sauer AG, Siegel RL, Jemal A, et al. Updated Review of Prevalence of Major Risk Factors and Use of Screening Tests for Cancer in the United States. Cancer Epidemiol Biomarkers Prev 2017;26:1192-208.

7. Pijanka JK, Stone N, Rutter AV, et al. Identification of different subsets of lung cells using Raman microspectroscopy and whole cell nucleus isolation. Analyst 2013;138:5052-8.

8. Farhane Z, Bonnier F, Casey A, et al. Cellular discrimination using in vitro Raman micro spectroscopy: the role of the nucleolus. Analyst 2015;140:5908-19.

9. Eberhardt K, Stiebing C, Matthäus C, et al. Advantages and limitations of Raman spectroscopy for molecular diagnostics: an update. Expert Rev Mol Diagn 2015;15:773-87.

10. Huser T, Chan J. Raman spectroscopy for physiological investigations of tissues and cells. Adv Drug Deliv Rev 2015;89:57-70.

11. Pilot R, Signorini R, Durante C, et al. A Review on Surface-Enhanced Raman Scattering. Biosensors (Basel) 2019;9:57.

12. Beć KB, Grabska J, Huck CW. Near-Infrared Spectroscopy in Bio-Applications. Molecules 2020;25:2948.

13. Buckley K, Ryder AG. Applications of Raman Spectroscopy in Biopharmaceutical Manufacturing: A Short Review. Appl Spectrosc 2017;71:1085-116.

14. Preferred reporting items for systematic review and metaanalysis protocols (PRISMA-P) 2015: elaboration and explanation. BMJ 2016;354:i4086.

15. Liang S, Chang L. Serum matrix metalloproteinase-9 level as a biomarker for colorectal cancer: a diagnostic metaanalysis. Biomark Med 2018;12:393-402.

16. Magee ND, Beattie JR, Carland C, et al. Raman microscopy in the diagnosis and prognosis of surgically resected nonsmall cell lung cancer. J Biomed Opt 2010;15:026015.

17. McGregor HC, Short MA, McWilliams A, et al. Realtime endoscopic Raman spectroscopy for in vivo early lung cancer detection. J Biophotonics 2017;10:98-110.

18. Gao F, Xiong Y, Zhang M, et al. Investigations on NIRSERS Spectra of Oxyhemoglobin for Lung Cancer Based on NIR-SERS Substrate. Guang Pu Xue Yu Guang Pu Fen Xi 2017;37:441-5.

19. Qian K, Wang Y, Hua L, et al. New method of lung cancer detection by saliva test using surface-enhanced Raman spectroscopy. Thorac Cancer 2018;9:1556-61.

20. Wang H, Zhang S, Wan L, et al. Screening and staging for non-small cell lung cancer by serum laser Raman spectroscopy. Spectrochim Acta A Mol Biomol Spectrosc 2018;201:34-8.

21. Moisoiu V, Stefancu A, Gulei D, et al. SERS-based differential diagnosis between multiple solid malignancies: breast, colorectal, lung, ovarian and oral cancer. Int J Nanomedicine 2019;14:6165-78.

22. Zhang K, Hao C, Huo Y, et al. Label-free diagnosis of lung cancer with tissue-slice surface-enhanced Raman spectroscopy and statistical analysis. Lasers Med Sci 2019;34:1849-55.

23. Zheng Q, Li J, Yang L, et al. Raman spectroscopy as a potential diagnostic tool to analyse biochemical alterations in lung cancer. Analyst 2020;145:385-92.

24. Bergholt MS, Zheng W, Lin K, et al. Raman endoscopy for in vivo differentiation between benign and malignant ulcers in the stomach. Analyst 2010;135:3162-8.

25. Keller MD, Vargis E, de Matos Granja N, et al. Development of a spatially offset Raman spectroscopy probe for breast tumor surgical margin evaluation. J Biomed Opt 2011;16:077006.

26. Huang $Z$, Teh SK, Zheng $W$, et al. In vivo detection of epithelial neoplasia in the stomach using image-guided Raman endoscopy. Biosens Bioelectron 2010;26:383-9.

27. Butler HJ, Ashton L, Bird B, et al. Using Raman spectroscopy to characterize biological materials. Nat Protoc 2016;11:664-87.

28. Smith ZJ, Huser TR, Wachsmann-Hogiu S. Raman scattering in pathology. Anal Cell Pathol (Amst) 2012;35:145-63.
Cite this article as: Chen $\mathrm{C}, \mathrm{Hao} \mathrm{J}$, Hao X, $\mathrm{Xu} \mathrm{W}$, Xiao C, Zhang J, Pu Q, Liu L. The accuracy of Raman spectroscopy in the diagnosis of lung cancer: a systematic review and metaanalysis. Transl Cancer Res 2021;10(8):3680-3693. doi: 10.21037/ tcr-21-515 